\title{
Tipos de violencia en la infancia que inciden en el abuso y dependencia de cannabis entre adolescentes: una revisión sistemática y metaanálisis
}

\author{
Influence of the type of childhood violence on \\ cannabis abuse and dependence among adolescents: \\ a systematic review and meta-analysis
}

\author{
Martínez-Mota Lucía*, Jiménez-Rubio Graciela*, Hernández-Hernández Olivia Tania*,**, \\ PÁez-Martínez Nayeli*,***. \\ * Dirección de Investigaciones en Neurociencias. Instituto Nacional de Psiquiatría Ramón de la Fuente Muñiz. Calzada México \\ Xochimilco 101, Col. San Lorenzo Huipulco, Tlalpan, CP 14370, Ciudad de México, México. \\ ** Consejo Nacional de Ciencia y Tecnología, Comisionada en el Instituto Nacional de Psiquiatría Ramón de la Fuente Muñiz. \\ Calzada México Xochimilco 101, Col. San Lorenzo Huipulco, Tlalpan, CP 14370, Ciudad de México, México. \\ *** Sección de Posgrado e Investigación. Escuela Superior de Medicina. Instituto Politécnico Nacional. Plan de San Luis y Díaz \\ Mirón, Col. Santo Tomás, Miguel Hidalgo, CP 11340, Ciudad de México, México.
}

\section{Resumen}

El uso recreativo de cannabis ha incrementado en todo el mundo, principalmente en la población adolescente. Se ha propuesto que la adversidad en la infancia contribuye al consumo de esta droga. El objetivo de esta revisión sistemática y metaanálisis fue analizar el efecto de diferentes tipos de violencia en la infancia sobre el consumo de cannabis en la adolescencia. Se realizó una búsqueda en diferentes bases de datos (PubMed, Science Direct, Web of Science, Ovid y CONRICyT) usando los términos de búsqueda: ("Cannabis" OR "Marijuana Smoking" OR "Marijuana Abuse") AND ("Child Abuse" OR "Domestic Violence" AND "Adolescent")), considerando todos los artículos publicados hasta el 3 de noviembre de 2017. Se calcularon los Odds Ratio (OR) del consumo de cannabis en adolescentes, para los diferentes tipos de abuso infantil, así como sus intervalos de confianza del $95 \%$ (IC 95\%). Se identificaron seis estudios, que incluyeron 10 843 adolescentes de uno u otro sexo. La asociación entre la violencia y el abuso/dependencia de cannabis en la adolescencia mostró los siguientes valores: abuso físico (OR: 1,58, IC $95 \%$ [1,01-2,46]), abuso sexual (OR: 2,35, IC 95\% [1,64-3,35]), y ser testigo de violencia (OR: 3,22, IC 95\% [0,63-16,54]). Los resultados muestran que el abuso sexual o físico durante etapas tempranas de la vida aumenta el riesgo de consumo de cannabis en la adolescencia. Los estudios que evaluaron otras formas de violencia fueron escasos. Los resultados destacan la importancia de diseñar programas integrales para reducir el uso y dependencia de cannabis mediante estrategias enfocadas a la prevención de la violencia en la infancia.

Palabras clave: Uso de cannabis; Abuso infantil; Infancia; Adolescencia; Violencia.

\begin{abstract}
The use of cannabis for recreational purposes has increased worldwide, and the proportion of cannabis users in the adolescent population is high. Susceptibility to cannabis use involves various factors, including childhood adversity; however, the effects of different types of violence on cannabis use have not been evaluated. The aim of this review was to analyze the effects of different types of violence on cannabis use in adolescence. We searched electronic databases (PubMed, Science Direct, Web of Science, Ovid and CONRICyT) using the following algorithm: (("Cannabis" OR "Marijuana Smoking” OR "Marijuana Abuse") AND ("Child Abuse" OR "Domestic Violence" AND "Adolescent")), considering all articles published up to November 3th, 2017. Odds ratios (ORs) were calculated for the effects of experiencing different types of violence during childhood on cannabis use. Six studies, which represented 10843 adolescents of both sexes, were ultimately included in the systematic review and meta-analysis. Three types of early-life adversity were associated with cannabis abuse/dependence: physical abuse (OR: 1.58, 95\% CI [1.01-2.46]), sexual abuse (OR: 2.35, 95\% CI [1.64-3.35]), and witnessing violence (OR: 3.22, 95\% CI [0.63-16.54]). The results indicated that two specific types of child maltreatment, sexual and physical abuse, were critical factors affecting vulnerability to cannabis use in adolescence. The number of studies examining other types of violence was limited. The results highlighted the importance of enhancing efforts to prevent violence, particularly sexual abuse, as part of integral programs designed to prevent cannabis abuse and dependence.

Keywords: Cannabis abuse; Child abuse; Childhood; Adolescents; Violence.
\end{abstract}




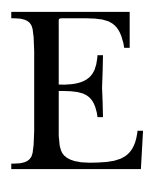

1 uso de cannabis con fines lúdicos ha aumentado a nivel mundial (United Nations Office on Drugs and Crime, 2015). En 1990 se reportaron once millones de casos de dependencia en todo el mundo, cifra que creció a trece millones en 2010 (Degenhardt et al., 2013). Podemos mencionar, por ejemplo, tasas de prevalencia para el uso de cannabis en la población general, de 15,2\% en la República Checa y de $13,7 \%$ en los Estados Unidos (Villatoro-Velázquez et al., 2012; United Nations Office on Drugs and Crime, 2015). De manera notable, la proporción de usuarios de cannabis en la población adolescente es particularmente elevada. La encuesta Youth Risk Behavior Survey (Encuesta sobre Conductas de Riesgo en la Juventud) de Estados Unidos reportó que, en estudiantes de preparatoria con edades entre 14 y 18 años, hubo un incremento en la tasa de prevalencia, pasando de $19,7 \%$ en 2007 a $23,4 \%$ en 2013 (Substance Abuse and Mental Health Services Administration, 2015).

En la adolescencia se observa un aumento en la conducta social, la toma de riesgos y la búsqueda de novedad. Este periodo de vida es crítico para el desarrollo cerebral, puesto que aumenta la velocidad y eficiencia de la comunicación entre neuronas, mediante mecanismos como la poda neuronal y el aumento de la mielinización (Spear, 2000; Spear, 2013). Se ha reportado que el uso de cannabis durante esta etapa de la vida puede causar alteraciones en la remodelación de la corteza cerebral y las conexiones neuronales, con la subsecuente alteración de la conducta (de la Fuente et al., 2015). Los usuarios de cannabis que inician el consumo durante la adolescencia tienen mayor probabilidad de presentar alteraciones en la memoria, la fluidez verbal, la toma de decisiones y la flexibilidad cognitiva (de la Fuente et al., 2015); además, el consumo crónico puede llevar a un deterioro de la inteligencia general, la memoria de corto plazo, las funciones ejecutivas, el juicio y la impulsividad motora (Meier et al., 2012; Ramaekers et al., 2006). De manera adicional, las consecuencias sociales derivadas del abuso de cannabis, durante la adolescencia tardía, se asocian con un desempeño académico deficiente y una carencia de oportunidades para obtener un empleo estable y construir una familia (Substance Abuse and Mental Health Services Administration, 2015).

Es importante hacer notar que el riesgo de iniciar el uso de cannabis y desarrollar dependencia difiere entre individuos. La etiología de estas diferencias involucra una combinación de factores biológicos, genéticos y medioambientales que pueden incrementar la vulnerabilidad (Buisman-Pijlman et al., 2014). Asimismo, el desarrollo de conductas de riesgo, tales como el uso y desarrollo de dependencia de cannabis, se han asociado con eventos que ocurren durante estados tempranos del desarrollo (es decir, la niñez y la adolescencia temprana) (Benjet, Borges y Medina-Mora, 2010). Esta es la razón primordial por la cual las intervenciones diseñadas para prevenir el uso de drogas deben enfocarse en los niños.

La adversidad en la infancia es altamente prevalente en todo el mundo (38,4\% a 39,1\%) e incluye: el abuso físico, el abuso sexual, la negligencia, la pobreza y la pérdida o separación de los padres (World Health Organization, 2016); además, los factores adversos se han asociado con un aumento en el riesgo para desarrollar desórdenes psiquiátricos (Kessler et al., 2010). El análisis de los datos estratificados por el tipo de adversidad muestra que la disfunción familiar y los abusos que causan daño corporal (por ejemplo, el abuso físico) son los predictores más robustos y confiables de psicopatologías en etapas posteriores de la vida, tales como el abuso de substancias y los trastornos externalizados (Benjet et al., 2010). El impacto de la adversidad a lo largo de la vida puede ser explicado por diferentes mecanismos, como la desregulación del sistema de estrés que produce alteraciones estructurales en el cerebro (Benjet et al., 2010), los mecanismos inadecuados de afrontamiento (Folkman y Lazarus, 1988; Folkman, Lazarus, Gruen y DeLongis, 1986) o la regulación emocional deficiente (Zimmermann et al., 2017).

Los niños están expuestos a la violencia en diferentes ámbitos, tanto en la comunidad, en la familia, como a tráves de los medios de comunicación (Osofsky, 1999). Esta exposición puede ocurrir de manera directa, en forma de victimización; o indirectamente, al presenciar algún tipo de violencia (Foster y Brooks-Gunn, 2009). La exposición a la violencia en la infancia resulta en índices más altos de desórdenes por estrés postraumático, depresión y problemas conductuales (Jester, Steinberg, Heitzeg y Zucker, 2015). Además, es un factor de riesgo para el desarrollo de abuso de sustancias en la adultez (Odds Ratio (OR) 2,84, IC 95\% [1,53-5,26]) (Benjet et al., 2010; Jester et al., 2015; Kuhar, 2012). De manera similar, el abuso sexual grave durante la infancia, se ha relacionado con un aumento en el riesgo para el abuso/dependencia al alcohol (OR 3,3, IC $95 \%[1,7-6,6])$, o el abuso/dependencia a otras drogas (OR 5,1, IC 95\% [2,5-10,2]) en la edad adulta temprana (Fergusson, Horwood y Lynskey, 1996). Así mismo, la historia de maltrato durante la infancia (incluyendo el abuso sexual, el abuso físico, el abuso emocional y la negligencia) se describe como un predictor importante del consumo de cannabis en adultos jóvenes (Vilhena-Churchill y Goldstein, 2014). A pesar de estos importantes hallazgos, no se cuenta con una evaluación sistemática que permita analizar de manera específica el tipo de violencia en la infancia y su impacto sobre el uso de cannabis en la adolescencia. Por lo anterior, el principal objetivo de esta revisión sistemática y metaanálisis fue examinar la relación entre la exposición a varios tipos de violencia en la infancia y el abuso o dependencia a cannabis en la adolescencia, además de determinar los principales factores de riesgo. 


\section{Metodología}

\section{Tipos de estudios}

Se incluyeron todos los estudios (casos y controles, estudios transversales y estudios longitudinales) que abordaron la relación entre la exposición a la violencia en la infancia y el uso de cannabis en la adolescencia. El abuso en la infancia (o violencia en la infancia) fue definido como la violencia perpetrada por los padres, los cuidadores primarios o los miembros de la comunidad. El abuso físico fue evaluado mediante preguntas respecto a ser golpeado, pateado, estrangulado, o atacado con una pistola, un cuchillo o con alguna otra arma, por cualquier persona; o respecto a haber sido golpeado por los padres hasta dejar daños corporales visibles. El abuso sexual fue definido y clasificado de acuerdo con distintos grados de aproximación sexual entre el adulto y el niño, y evaluado con preguntas acerca de episodios sin contacto físico (incluyendo posturas indecentes, masturbación pública, propuestas sexuales), o incidentes que involucraron intención de contacto, o relaciones sexuales consumadas. El ser testigo de violencia fue definido por la exposición a la violencia dirigida contra otro miembro de la familia o cualquier persona, y en cualquier ambiente (casa o comunidad). Este tipo de violencia fue evaluado con preguntas acerca de haber presenciado bofetadas, golpes, patadas, o sujeción forzada entre los padres, o el haber presenciado amenazas hacia cualquier persona con un cuchillo, una pistola o alguna otra arma. La adolescencia fue definida como el periodo de vida entre los 12 y 17 años. El uso (o abuso) de cannabis incluyó todas las formas de consumo, abarcando el uso en una sola ocasión, el uso no frecuente o intermitente, o el uso crónico. Se excluyeron todos los estudios en los cuales el consumo de cannabis no se reportó en la adolescencia o se experimentó vía prenatal.

\section{Participantes}

Tomamos en cuenta a los adolescentes que fueron incorporados a las muestras de estudios nacionales, y cuyos datos fueron sometidos a diferentes tipos de análisis, de acuerdo con el objetivo del estudio original. Todos los participantes dieron su consentimiento informado previo al inicio de la entrevista y en todos los reportes se mencionó que los Comités de Evaluación Institucional aprobaron los respectivos estudios.

\section{Tipos de exposición}

Se incluyeron todos los trabajos que analizaron la exposición de todos los tipos de violencia hacia los niños y que fue perpetrada por adultos.

\section{Evaluación de resultados}

El desenlace principal fue el uso o abuso de cannabis durante la adolescencia; además, donde fue posible, se realizó un análisis considerando las diferencias por sexo de los participantes.
Métodos de búsqueda para la identificación de estudios

La identificación de los artículos se realizó a través de la exploración en las siguientes bases de datos: PubMed (MEDLINE), Science Direct, Web of Science, Ovid (MEDLINE), y CONRICyT (Consorcio Nacional de Recursos de Información Científica y Tecnológica-México). Incluimos todos los trabajos que se llevaron a cabo antes del 3 de noviembre de 2017 y que se obtuvieron con la búsqueda a través de los siguientes términos y operadores booleanos: ( («Cannabis» OR «Marijuana Smoking» OR «Marijuana Abuse») AND («Child Abuse» OR «Domestic Violence» AND «Adolescent»)). Los términos de búsqueda para Cannabis fueron: Marihuana; Marijuana; Hashish; Cannabis sativa. Los términos para el abuso de mariguana incluyeron: Cannabis-related Disorder; Cannabis Abuse; Marijuana Dependence; Marihuana Abuse; Hashish Abuse. Los términos para fumar mariguana fueron: Marihuana Smoking; Hashish Smoking; Cannabis Smoking. El abuso infantil tuvo como sinónimos: Abuse, Child; Child Mistreatment; Mistreatment, Child; Child Maltreatment; Maltreatment, Child; Child Neglect; Neglect, Child. Los términos para la violencia doméstica incluyeron: Violence, Domestic; Family Violence; Violence, Family. No hubo restricciones respecto al idioma o el estado de la publicación.

\section{Búsqueda a través de otras fuentes}

Las listas de referencias de todos los artículos en texto completo fueron revisadas para identificar nuevos estudios, los cuales no se obtuvieron a través de las bases de datos.

\section{Recopilación de datos}

Dos autores realizaron, de manera independiente, la revisión de los títulos, los resúmenes y los textos completos potencialmente relevantes; todo esto de acuerdo con los criterios de inclusión y exclusión previamente establecidos. Los desacuerdos se resolvieron a través de una discusión o mediante la consulta con un tercer juez (Figura 1).

Se diseñó un formato para extraer la información relevante de cada uno de los artículos. Los datos obtenidos abarcaron: el nombre del primer autor, el año de publicación, el tipo de estudio, los datos de los participantes, el tamaño de la muestra, el tipo de abuso en la infancia, el uso de cannabis en la adolescencia, los hallazgos de los estudios, y los ORs. En primera instancia, los hallazgos más importantes se sintetizaron de forma descriptiva, y posteriormente se consideró la factibilidad para realizar un metaanálisis.

\section{Estadística}

En los estudios de casos y controles y los estudios transversales la medición de los efectos y la evaluación de la heterogeneidad de los OR con IC 95\% fueron calculados considerando desenlaces dicotómicos (por ejemplo, uso de cannabis vs no uso de cannabis). Para los estudios lon- 
gitudinales (por ejemplo, cohortes) fue calculado el riesgo relativo. Los resultados de los análisis se presentan como diagramas de bosque.

La heterogeneidad estadística de cada metaanálisis fue evaluada usando $\mathrm{I}^{2}$ y $\chi^{2}$, así como los valores de P. Consideramos que existía heterogeneidad importante si los valores de $\mathrm{I}^{2}$ fueron mayores del $50 \%$. Los datos se analizaron usando modelos de efectos aleatorios o de efectos fijos según correspondiera.

\section{Sintesis de datos}

Los datos se analizaron usando el Review Manager Software (RevMan) en su versión 5.3.

\section{Resultados}

La búsqueda de la literatura aportó 190 artículos, de los cuales 13 fueron eliminados por estar duplicados. De los 177 restantes, 135 fueron excluidos a partir de la lectura de los resúmenes. Se realizó la lectura de 42 textos completos, de los cuales 36 fueron eliminados y únicamente 6 artículos cumplieron en su totalidad con los criterios de inclusión descritos en los métodos (Figura 1).

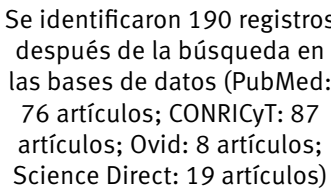

Se identificaron 190 registros después de la búsqueda en las bases de datos (PubMed: 76 artículos; CONRICYT: 87 artículos; Ovid: 8 artículos; Science Direct: 19 artículos)

Se eligieron 177 registro después de eliminar los artículos duplicados

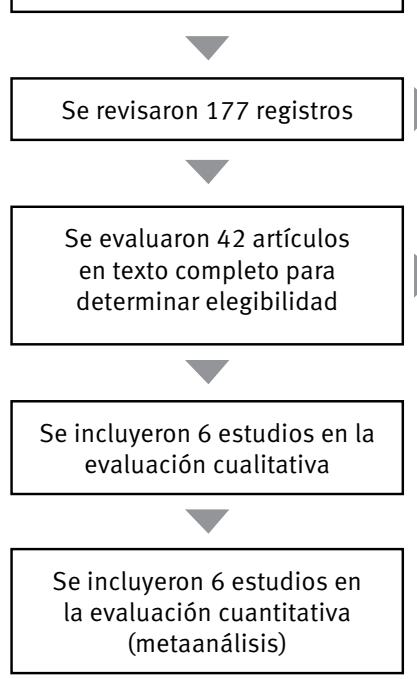

Figura 1. Diagrama de flujo de la identificación y selección de los estudios para la revisión sistemática.

\section{Descripción de los estudios}

La búsqueda en las bases de datos incluyó todos los tipos de violencia, no obstante, únicamente seis artículos informaron las frecuencias de casos de individuos con exposición a la violencia (abuso sexual, abuso físico, y ser testigo de violencia) durante la infancia y uso o abuso de cannabis en la adolescencia. Cinco estudios fueron diseños de cohorte, pero con evaluaciones transversales efectuadas en periodos concretos de la vida de los participantes (Dubowitz et al., 2016; Duncan et al., 2008; Fergusson y Horwood, 1998; Fergusson y Lynskey, 1997; Sartor et al., 2015), mientras que el estudio de Kilpatrick et al. (2000) fue desarrollado a partir de una muestra probabilística derivada de la National Household Survey (Encuesta Nacional en Hogares). Los estudios conducidos por Fergusson y Horwood (1998) y Fergusson y Lynskey (1997), fueron desarrollados a partir de la misma muestra; sin embargo, para los fines de esta revisión sistemática, los datos fueron tratados de forma independiente ya que son resultado de diferentes tipos de violencia (Tabla 1). Los estudios de cohorte reportaron varias evaluaciones de seguimiento, pero no fueron comparables entre estudios, por lo que, para fines del metaanálisis consideramos únicamente los datos transversales.

Los estudios realizados por Fergusson y Horwood (1998), y Fergusson y Lynskey (1997) fueron desarrollados en Nueva Zelanda (Fergusson, Horwood, Shannon, y Lawton, 1989), en tanto que los trabajos de Kilpatrick et al. (2000), Duncan et al. (2008), Sartor et al. (2015) y Dubowitz et al. (2016) tuvieron lugar en los Estados Unidos.

Tres estudios analizaron la exposición a la violencia en la infancia y las características del abuso mediante entrevistas estructuradas o cuestionarios (Dubowitz et al., 2016; Fergusson y Horwood, 1998; Fergusson y Lynskey, 1997). Por su parte, Dubowitz et al. (2016) recopilaron datos a partir de varias fuentes: informes de los Servicios de Protección al Menor, y posteriormente, de entrevistas a los padres y los niños. Un estudio recopiló la información mediante una entrevista telefónica diseñada por los autores (Kilpatrick et al., 2000), mientras que dos estudios investigaron vía telefónica las características del abuso con la Semi-structured Assessment for the Genetics of Alcoholism (SSAGA; Evaluación Semiestructurada para el Estudio de la Heredabilidad del Alcoholismo) (Duncan et al., 2008; Sartor et al., 2015) (Tabla 2). Por lo tanto, los estudios analizados en esta revisión reportaron una variedad de métodos para la obtención de la información relativa a los diferentes tipos de violencia.

Cuatro estudios identificaron que los participantes sufrieron abuso físico (Dubowitz et al., 2016; Duncan et al., 2008; Fergusson y Lynskey, 1997; Kilpatrick et al., 2000), mientras que el de Dubowitz et al. (2016) informó además sobre otras formas de abuso, tales como negligencia y abuso emocional. Con excepción del reporte de Fergusson y Lynskey (1997), todos los artículos mostraron información 
Tabla 1. Características de los estudios epidemiológicos de la exposición infantil a la violencia sobre el uso, consumo o dependencia a cannabis.

\begin{tabular}{|c|c|c|c|c|c|c|c|c|c|c|}
\hline $\begin{array}{l}\text { Autores, } \\
\text { año }\end{array}$ & País & Estudio & TE & $\begin{array}{l}\text { Definición } \\
\text { de expo- } \\
\text { sición }\end{array}$ & $\begin{array}{l}\text { Instrumento/ } \\
\text { Evaluación }\end{array}$ & $\begin{array}{l}\text { Resultado/ } \\
\text { Diagnóstico }\end{array}$ & $\begin{array}{l}\text { Tamaño de la } \\
\text { muestra/ Datos } \\
\text { demográficos }\end{array}$ & $\begin{array}{c}\text { Edad de } \\
\text { exposición }\end{array}$ & $\begin{array}{l}\text { Edad durante } \\
\text { la evaluación }\end{array}$ & Estimación/Nota \\
\hline $\begin{array}{l}\text { Fergusson } \\
\text { y Lynskey, } \\
1997\end{array}$ & NZ & CHDS & $\mathrm{T}$ & $\mathrm{AF}$ & E C-a-C, E, C & $\begin{array}{c}\text { Abuso o } \\
\text { dependencia } \\
\text { de Cannabis } \\
\text { DSM-IV }\end{array}$ & $\begin{array}{l}\mathrm{N}=1265 \\
\text { Hombres y } \\
\text { mujeres }\end{array}$ & «16 años & «18 años & $\begin{array}{l}\text { Tasa de abuso de } \\
\text { sustancias por grado } \\
\text { de violencia ejercida } \\
\text { por los padres }\end{array}$ \\
\hline $\begin{array}{l}\text { Fergusson } \\
\text { y Horwood, } \\
1998\end{array}$ & $\mathrm{NZ}$ & CHDS & $\mathrm{T}$ & $\begin{array}{l}\text { Al } \\
\text { AS }\end{array}$ & E C-a-C, E, C & $\begin{array}{c}\text { Abuso o } \\
\text { dependencia } \\
\text { de Cannabis } \\
\text { DSM-IV }\end{array}$ & $\begin{array}{l}\mathrm{N}=1265 \\
\text { Hombres y } \\
\text { mujeres }\end{array}$ & «16 años & «18 años & $\begin{array}{l}\text { Tasa de abuso de } \\
\text { sustancias por grado } \\
\text { de violencia entre los } \\
\text { padres }\end{array}$ \\
\hline $\begin{array}{l}\text { Kilpatrick et } \\
\text { al., } 2000\end{array}$ & EE.UU & NSA & $\mathrm{T}$ & $\begin{array}{l}\text { AGF } \\
\text { AGS } \\
\text { TV }\end{array}$ & $\begin{array}{c}\text { ET } \\
\text { estructurada }\end{array}$ & $\begin{array}{c}\text { Abuso o } \\
\text { dependencia } \\
\text { de Cannabis } \\
\text { DSM-IV }\end{array}$ & $\begin{array}{l}\mathrm{N}=3907 \\
\text { Hombres y } \\
\text { mujeres }\end{array}$ & $\begin{array}{c}\text { Año } \\
\text { anterior }\end{array}$ & 12-17 años & $\begin{array}{l}\text { AF: OR 4,84 } \\
\text { AS: OR } 3,80 \\
\text { TV: OR } 8,42 \\
\text { IC } 95 \% \text { no } \\
\text { informados }\end{array}$ \\
\hline $\begin{array}{l}\text { Duncan et } \\
\text { al., } 2008\end{array}$ & EE.UU & VETR & $\mathrm{T}$ & $\begin{array}{l}\mathrm{AS} \\
\mathrm{AF}\end{array}$ & ET-SSAGA & $\begin{array}{c}\text { Abuso o } \\
\text { dependencia } \\
\text { de Cannabis } \\
\text { DSM-IV }\end{array}$ & $\begin{array}{l}\mathrm{N}=819 \\
\text { Hombres y } \\
\text { mujeres }\end{array}$ & «16 años & $\begin{array}{c}\text { Adolescentes } \\
\text { o adultos } \\
\text { jóvenes }\end{array}$ & $\begin{array}{l}\text { RR } 2,16 \\
(\text { IC } 95 \% 1,48-3,16)\end{array}$ \\
\hline $\begin{array}{l}\text { Sartor et } \\
\text { al., } 2015\end{array}$ & EE.UU & $\begin{array}{l}\text { MOAFTS } \\
\text { MOFAM }\end{array}$ & $\mathrm{T}$ & AS & ET-SSAGA & $\begin{array}{c}\text { Abuso o } \\
\text { dependencia } \\
\text { de Cannabis } \\
\text { DSM-IV }\end{array}$ & $\begin{array}{l}\mathrm{N}=4150 \\
\text { Mujeres, } \\
\text { gemelos y } \\
\text { hermanos }\end{array}$ & «16 años & 18-29 años & $\begin{array}{l}\text { Europeo Americano } \\
\text { RR } 1,57 \text { (IC } 95 \% \\
1,37-1,79) \\
\text { Afroamericano } \\
\text { RR 2,52 (IC } 95 \% \\
1,52-4,18)\end{array}$ \\
\hline $\begin{array}{l}\text { Dubowitz et } \\
\text { al., } 2016\end{array}$ & EE.UU & $\begin{array}{l}\text { LONG- } \\
\text { SCAN }\end{array}$ & $\mathrm{T}$ & $\begin{array}{c}\mathrm{AS} \\
\mathrm{AF} \\
\mathrm{N} \\
\mathrm{ME}\end{array}$ & MMCS & $\begin{array}{l}\text { Respuesta } \\
\text { dicotómica: } \\
\text { No uso de } \\
\text { cannabis } \\
\text { o uso de } \\
\text { cannabis }\end{array}$ & $\begin{array}{c}\mathrm{N}=702 ; \\
332 \text { hombres y } \\
370 \text { mujeres }\end{array}$ & $\begin{array}{c}\text { Desde el } \\
\text { nacimiento } \\
\text { hasta los } \\
18 \text { años }\end{array}$ & 18 años & $\begin{array}{l}\text { ME: OR } 1,32 \text { (IC } 95 \% \\
0,98-1,78 \\
\text { N: OR } 0,78 \\
\text { (IC } 95 \% 0,49-1,25)\end{array}$ \\
\hline
\end{tabular}

Nota. NZ: Nueva Zelanda; EE.UU: Estados Unidos; CHDS: Christchurch Health \& Development Study (Estudio de Salud y Desarrollo en Christchurch); NSA: National Survey of Adolescents (Encuesta Nacional en Adolescentes); VETR: The Vietnam Era Twin Registry (Registro de Gemelos de la Era de Vietnam; registros de descendencia); MOAFTS: Missouri Adolescent Female Twin Study (Estudio de Gemelas Adolescentes de Missouri); MOFAM: Missouri Family Study (Estudio de Familias en Missouri); LONGSCAN: Longitudinal Studies of Child Abuse and Neglect (Estudios Longitudinales sobre el Abuso y Negligencia en la Infancia); E C-a-C: Evaluación cara-a-cara, para padres e hijos; obtenido de Fergusson et al., 1989; TE: Tipo de estudio; T: Evaluación transversal; E: Entrevista; C: Cuestionario; AF: Abuso físico; Al: Abuso intrafamiliar; AS: Abuso sexual; AGF: Agresión Física; AGS: Agresión sexual; TV: Testigo de violencia; N: Negligencia; ME: Maltrato emocional; SSAGA: Semi-structured Assessment for the Genetics of Alcoholism (Evaluación Semiestructurada para el Estudio de la Heredabilidad del Alcoholismo); ET; Entrevista telefónica; MMCS: Maltreatment Coding Scheme (Programa de Codificación del Maltrato) versión modificada de Barnett et al., 1993; DSM: Manual Diagnóstico y Estadístico de los Trastornos Mentales 4a edición. OR: Odds Ratio; IC: Intervalo de confianza; RR: Riesgo relativo.

sobre la ocurrencia de abuso sexual a niños; sin embargo, solo cuatro artículos proporcionaron datos para integrar al análisis estadístico. A su vez, dos artículos mostraron datos de la exposición indirecta a la violencia, es decir, cuando los niños fueron testigos de encuentros violentos entre padres, parejas, etc. (Fergusson y Horwood, 1998; Kilpatrick et al., 2000). Duncan et al. (2008) y Kilpatrick et al. (2000) incluyeron en su entrevista tres y seis preguntas, respectivamente, relacionadas al abuso sexual. El número de preguntas sobre el abuso físico fluctuó entre tres y diez. Respecto a la exposición indirecta a la violencia (ser testigo), Kilpatrick et al. (2000) hicieron una pregunta general, mientras que Fergusson y Horwood (1998) indagaron las características de este tipo de abuso (i.e., exploración a profundidad sobre el tipo y frecuencia de los incidentes) mediante ocho preguntas. Dubowitz y colaboradores (2016) siguieron el protocolo del proyecto Longitudinal Studies of Child Abuse and Neglect (LONGSCAN; Estudios Longitudinales sobre el Abuso y Negligencia en la Infancia) (English, Bangdiwala y Runyan, 2005; Runyan et al., 1998) que consistió en obtener datos de cinco formas de abuso, a partir de reportes del Servicio de Protección al Menor, así como de entrevistas a los padres y los niños, que fueron categorizadas de acuerdo con el Maltreatment Coding Scheme (MMCS; Programa de Codificación del Maltrato) (Barnett, Manly y Cicchetti, 1993) (Tabla 1).

Para la obtención del desenlace o resultado principal -abuso o dependencia a cannabis- se preguntó sobre el consumo en cualquier momento antes de los 18 años, o durante el año que precedió a la entrevista. Aunque esta evaluación se llevó a cabo por métodos heterogéneos (Tabla 2), los estudios coincidieron en el uso de los criterios diagnósticos para el Desorden por Abuso de Sustancias, establecidos en el Manual Diagnóstico y Estadístico de los Trastornos Mentales en su versión 4 (DSM-IV, American Psychiatric Association, 1994), como fundamento para la 
Tipos de violencia en la infancia que inciden en el abuso y dependencia de cannabis entre adolescentes: una revisión sistemática y metaanálisis

Tabla 2. Ítems usados para definir abuso en la infancia y abuso o dependencia a cannabis.

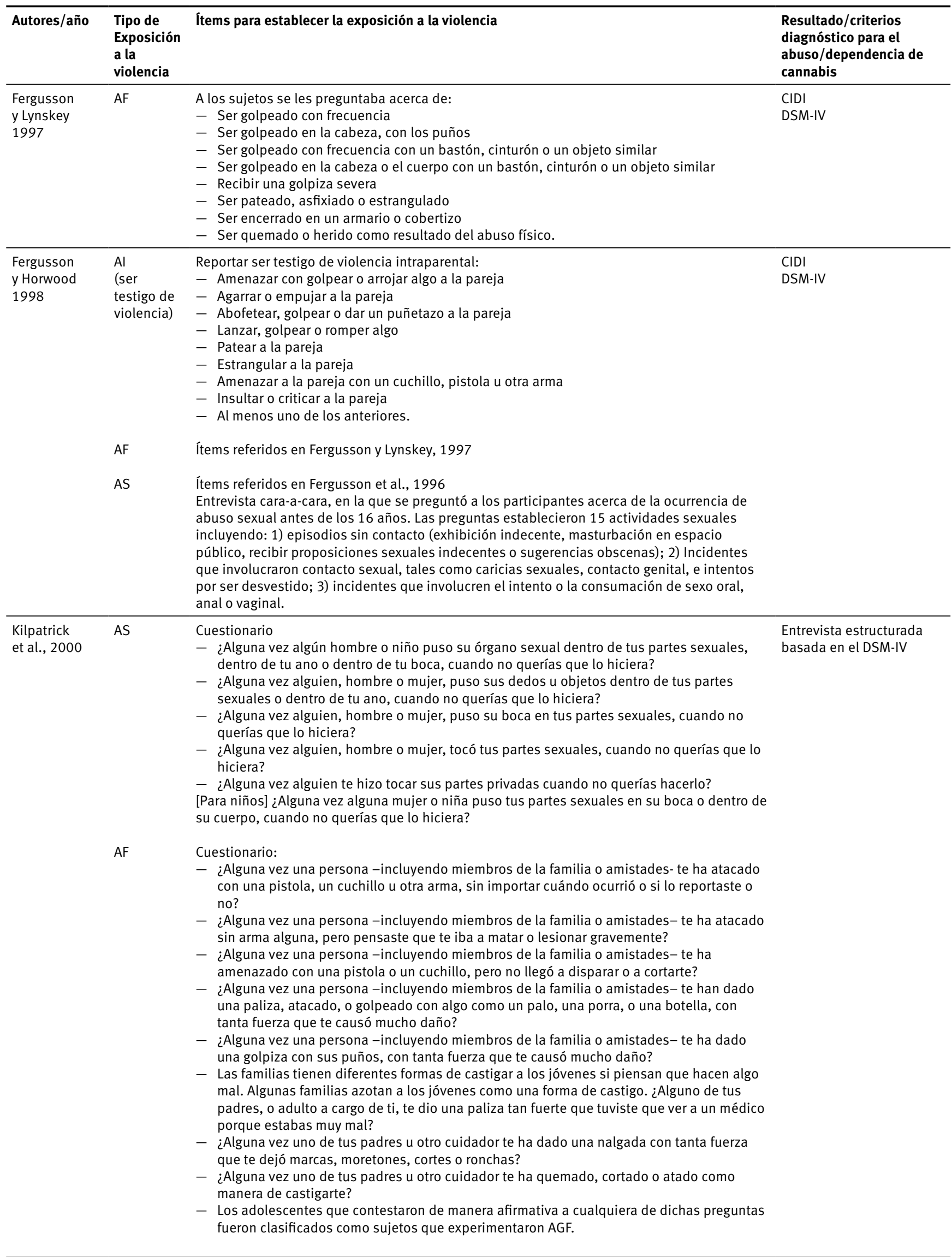




\begin{tabular}{|c|c|c|c|}
\hline $\begin{array}{l}\text { Duncan } \\
\text { et al., } 2008\end{array}$ & $\mathrm{AF}$ & $\begin{array}{l}\text { Se consideró que una persona experimentó AF durante su infancia (6-12 años) cuando: } \\
\text { a) reportó AF antes de la edad de } 16 \text { años en la entrevista sobre eventos traumáticos, b) } \\
\text { contestó “sí” a “¿Cuando tenías entre } 6 \text { y } 12 \text { años, algún adulto te causó daño físico o te } \\
\text { lesionó a propósito?” c) reportó “con frecuencia” "recibir puñetazos o ser golpeado con un } \\
\text { cinturón, palo u otro objeto similar por parte de su madre o padre”. } \\
\text { Se consideró que ocurrió AS cuando una persona reportó haber sido: a) violado o b) } \\
\text { experimentado AS antes de la edad de } 16 \text { años o c) forzado a tener sexo antes de la edad de } \\
16 \text { años. }\end{array}$ & $\begin{array}{l}\text { Entrevista estructurada } \\
\text { basada en el DSM-IV. } \\
\text { Diagnóstico de abuso, } \\
\text { cuando la persona } \\
\text { reportó cualquier de } \\
\text { los cuatro abusos de } \\
\text { cannabis según el DSM-IV. } \\
\text { Dependencia, si la } \\
\text { persona reportó tres o } \\
\text { más de los siete síntomas } \\
\text { de dependencia a } \\
\text { cannabis, incluyendo } \\
\text { abstinencia. }\end{array}$ \\
\hline $\begin{array}{l}\text { Sartor } \\
\text { et al., } 2015\end{array}$ & AS & $\begin{array}{l}\text { Primer cuestionario: Reporte de haber sido violado o abusado sexualmente antes de los } 15 \\
\text { años } \\
\text { Segundo cuestionario: ¿Alguna persona te ha obligado a tener sexo antes de que tuvieras } 15 \\
\text { años? } \\
\text { Tercer cuestionario: Antes de que cumplieras los } 16 \text { años, ¿algún miembro de tu familia te } \\
\text { forzó a tener contacto sexual? Antes de que cumplieras los } 16 \text { años, ¿alguna persona (que no } \\
\text { era de la familia) que tenía } 5 \text { o más años que tú, te forzó a tener contacto sexual? }\end{array}$ & $\begin{array}{l}\text { Entrevista } \\
\text { semiestructurada } \\
\text { basada en el DSM-IV. } \\
\text { Personas que reportaron } \\
\text { uno o más de los síntomas } \\
\text { de abuso o dependencia } \\
\text { a cannabis. }\end{array}$ \\
\hline $\begin{array}{l}\text { Dubowitz } \\
\text { et al., } 2016\end{array}$ & AS & $\begin{array}{l}\text { A los sujetos se les preguntó acerca de: } \\
\text { Exposición, explotación, abuso o penetración. } \\
\text { Si alguna vez han sido golpeados en: } \\
\text { Cabeza, torso, nalgas o extremidades. } \\
\text { Si alguna vez han sido: } \\
\text { Sacudidos con violencia, estrangulados, quemados. }\end{array}$ & $\begin{array}{l}\text { Respuesta dicotómica: } \\
\text { Ningún uso de cannabis o } \\
\text { uso de cannabis. }\end{array}$ \\
\hline
\end{tabular}

Nota. AF: Abuso físico; AS: Abuso sexual; AI: Abuso intraparental; CIDI: Composite International Diagnostic Interview (Entrevista Diagnóstica Internacional Compuesta). DSM-IV: Diagnostic and Statistical Manual of Mental Disorders 4 a edición (Manual Diagnóstico y Estadístico de los Trastornos Mentales, $4^{\text {a }}$ edición).

elaboración de sus cuestionarios. Dos estudios utilizaron la WHO-CIDI (World Health Organization Composite International Diagnostic Interview; Cottler y Compton, 1993), que es de tipo 'cara-a-cara', y otros tres trabajos emplearon entrevistas telefónicas. Por su parte, Dubowitz et al. (2016), identificó el uso de cannabis por medio de una pregunta dicotómica realizada a los participantes a las edades de 12 , 14, 16 y 18 años. Cuando ellos respondieron afirmativamente, entonces fueron cuestionados acerca de la frecuencia de uso (frecuente u ocasional).

El análisis de los posibles sesgos en la investigación mostró que el estudio de Kilpatrick et al. (2000) seleccionó una muestra representativa de la comunidad, mientras que los otros cinco estudios no hicieron una selección aleatoria de la muestra. Dado que la mayoría de los estudios fueron cohortes, no se establecieron procedimientos de ocultación o cegado, de hecho, en estos estudios de seguimiento la población fue evaluada en repetidas ocasiones, culminando con la investigación cuando los participantes alcanzaron la edad de 18 años. Los autores de los seis artículos reportaron la tasa de deserción de los participantes, aunque, destacaron un nivel aceptable de cumplimiento de los participantes para asistir a las entrevistas 'cara-a-cara' o contestar las llamadas telefónicas. El rango de edad a la que ocurrió el abuso fue descrito en todos los reportes: cuatro estudios informaron que los participantes tenían menos de 16 años cuando se llevó a cabo el hecho, uno informó que el abuso se presentó el año anterior a la evaluación, y otro documentó que el abuso sucedió entre el nacimiento y los 18 años. El periodo en el cual se efectuó la evaluación del desenlace varió entre estudios, con excepción de las investigaciones de Fergusson y Horwood (1998), y Fergusson y Lynskey (1997), que se llevaron a cabo con la misma población. La validez de las evaluaciones a través de una entrevista telefónica fue un motivo de preocupación entre los investigadores, ya que los mismos autores reconocen que no tienen evidencia de una comparación de sus resultados contra otros de la literatura, obtenidos mediante procedimientos diferentes (Kilpatrick et al., 2000). A su vez, se desconoce si los adolescentes fueron veraces al informar sobre sus antecedentes de abuso y hábitos de consumo de cannabis durante la entrevista telefónica (Kilpatrick et al., 2000; Duncan et al., 2008; Sartor et al., 2015). En general, se observó un nivel de sesgo alto entre los estudios analizados, derivado de la variabilidad de los procedimientos de muestreo y evaluación del desenlace, así como por la posible modulación de otras variables que podrían incidir en el desenlace, tales como, la presencia de comorbilidades psiquiátricas o el abuso de sustancias distintas a cannabis.

\section{Población estudiada}

Obtuvimos un total de 10843 participantes (3 395 hombres y 7448 mujeres) a partir de los seis estudios seleccionados. Efectuamos el metaanálisis con el número de casos de individuos expuestos a la violencia y que consumieron o no cannabis, así como el número de sujetos que no estuvieron expuestos a la violencia, y que consumieron o no cannabis. Los tipos de violencia analizados fueron el abuso sexual, el abuso físico y ser testigo de violencia. 
A

\begin{tabular}{lccccccc}
\hline & \multicolumn{2}{c}{ Abuso físico } & \multicolumn{2}{c}{ No abuso físico } & & Odds Ratio \\
\cline { 2 - 5 } Estudio o subgrupo & Eventos & Total & Eventos & Total & Ponderaciones & M-H, Aleatorio, Cl 95\% \\
\hline Dubowitz et al., 2016 & 226 & 389 & 147 & 313 & $28,6 \%$ & $1,57[1,16 ; 2,11]$ \\
Duncan et al., 2008 & 30 & 105 & 158 & 714 & $24,4 \%$ & $1,41[0,89 ; 2,23]$ \\
Fergusson y Lynskey, 1997 & 11 & 111 & 110 & 914 & $19,3 \%$ & $0,80[0,42 ; 1,55]$ \\
Kilpatrick et al., 2000 & 83 & 940 & 64 & 1912 & $27,7 \%$ & $2,80[2,00 ; 3,91]$ \\
\hline Total (IC 95\%) & & 1545 & & 3853 & $100,0 \%$ & $1,58[1,01 ; 2,46]$ \\
Eventos totales & 350 & & 479 & & &
\end{tabular}

Heterogeneidad: $\operatorname{Tau}^{2}=0,16 ; \mathrm{Chi}^{2}=14,32, \mathrm{gl}=3(\mathrm{P}=0,002) ; \mathrm{I}^{2}=79 \%$

Test para el efecto global: $Z=1,99(P=0,05)$

B

\begin{tabular}{|c|c|c|c|c|c|c|}
\hline \multirow[b]{2}{*}{ Estudio o subgrupo } & \multicolumn{2}{|c|}{ Abuso sexual } & \multicolumn{2}{|c|}{ No abuso sexual } & \multirow[b]{2}{*}{ Ponderaciones } & \multirow{2}{*}{$\frac{\text { Odds Ratio }}{\mathrm{M}-\mathrm{H} \text {, Aleatorio, } \mathrm{Cl} 95 \%}$} \\
\hline & Eventos & Total & Eventos & Total & & \\
\hline Dubowitz et al., 2016 & 118 & 196 & 258 & 506 & $25,8 \%$ & $1,45[1,04 ; 2,03]$ \\
\hline Duncan et al., 2008 & 30 & 70 & 158 & 749 & $20,0 \%$ & $2,81[1,69 ; 4,65]$ \\
\hline Kilpatrick et al., 2000 & 35 & 327 & 112 & 3580 & $23,6 \%$ & $3,71[2,49 ; 5,53]$ \\
\hline Sartor et al., 2015 & 406 & 589 & 1790 & 3561 & $30,6 \%$ & $2,20[1,82 ; 2,64]$ \\
\hline Total (IC 95\%) & & 1182 & & 8396 & $100,0 \%$ & $2,35[1,64 ; 3,35]$ \\
\hline Eventos totales & 589 & & 2318 & & & \\
\hline \multicolumn{7}{|c|}{ Heterogeneidad: $\mathrm{Tau}^{2}=0,10 ; \mathrm{Chi}^{2}=13,51, \mathrm{gl}=3(\mathrm{P}=0,004) ; \mathrm{I}^{2}=78 \%$} \\
\hline \multicolumn{7}{|c|}{ Test para el efecto global: $Z=4,70(P=0,00001)$} \\
\hline
\end{tabular}

C

\begin{tabular}{lccccccc}
\hline & \multicolumn{2}{c}{$\begin{array}{c}\text { Testigo de } \\
\text { violencia }\end{array}$} & \multicolumn{2}{c}{$\begin{array}{c}\text { No testigo de } \\
\text { violencia }\end{array}$} & & Odds Ratio \\
\cline { 2 - 5 } \cline { 5 - 6 } Estudio o subgrupo & Eventos & Total & Eventos & Total & Ponderaciones & M-H, Aleatorio, Cl 95\% \\
\hline Fergusson y Lynskey, 1997 & 57 & 121 & 353 & 914 & $50,2 \%$ & $1,42[0,97 ; 2,07]$ \\
Kilpatrick et al., 2000 & 123 & 147 & 1540 & 3760 & $49,8 \%$ & $7,39[4,75 ; 11,50]$ \\
\hline Total (IC 95\%) & 180 & 268 & & 4674 & $100,0 \%$ & $3,22[0,63 ; 16,54]$ \\
Eventos totales & & 1893 & & & \\
\hline
\end{tabular}

Heterogeneidad: $\mathrm{Tau}^{2}=1,35 ; \mathrm{Chi}^{2}=31,44, \mathrm{gl}=1(\mathrm{P}=0,00001) ; \mathrm{I}^{2}=97 \%$

Test para el efecto global: $Z=1,40(P=0,16)$

D

\begin{tabular}{|c|c|c|c|c|c|c|}
\hline \multirow[b]{2}{*}{ Estudio o subgrupo } & \multicolumn{2}{|c|}{ Abuso sexual } & \multicolumn{2}{|c|}{ No abuso sexual } & \multirow[b]{2}{*}{ Ponderaciones } & \multirow{2}{*}{$\begin{array}{c}\text { Odds Ratio } \\
\text { M-H, Fijo, Cl 95\% }\end{array}$} \\
\hline & Eventos & Total & Eventos & Total & & \\
\hline Duncan et al., 2008 & 18 & 70 & 37 & 324 & $5,8 \%$ & $2,69[1,42 ; 5,07]$ \\
\hline Sartor et al., 2015 & 406 & 589 & 1790 & 3561 & $94,2 \%$ & $2,20[1,82 ; 2,64]$ \\
\hline Total (IC 95\%) & & 659 & & 3885 & $100,0 \%$ & $2,22[1,86 ; 2,66]$ \\
\hline Eventos totales & 424 & & 1827 & & & \\
\hline \multicolumn{7}{|c|}{ Heterogeneidad: $\mathrm{Chi}^{2}=0,36 ; \mathrm{gl}=1(\mathrm{P}=0,55) ; \mathrm{I}^{2}=0 \%$} \\
\hline \multicolumn{7}{|c|}{ Test para el efecto global: $Z=8,73(P=0,00001)$} \\
\hline
\end{tabular}

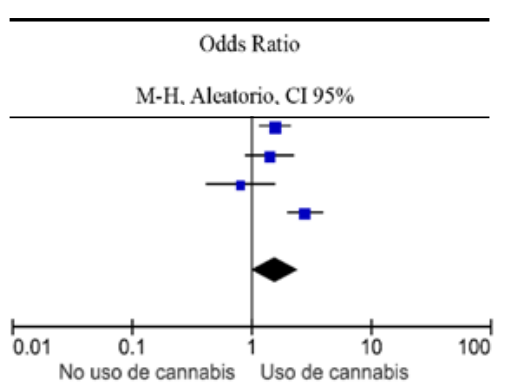

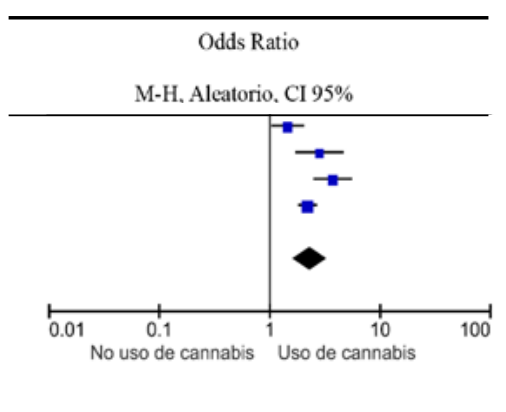

$$
\text { . }
$$




\section{Efecto de la exposición a la violencia}

El análisis estadístico mostró que los tres tipos de violencia contribuyeron a incrementar el riesgo para el uso de cannabis, o el desarrollo de dependencia a cannabis en la adolescencia, aunque dicha asociación varió de acuerdo con el tipo de abuso (abuso físico: OR: 1,58, IC 95\% [1,012,46], Figura 2, panel A; abuso sexual: OR: 2,35, IC 95\% [1,64-3,35], Figura 2, panel B; ser testigo de violencia: OR: 3,22, IC 95\% [0,63-16,54], Figura 2, panel C). Los OR y los IC indicaron que, en los participantes de uno u otro sexo, el riesgo para el uso o dependencia de cannabis se produjo en mayor medida cuando los adolescentes sufrieron abuso sexual en una etapa temprana de la vida, seguida por el abuso físico. Algunos artículos no especificaron la frecuencia del desenlace de acuerdo con el sexo de los participantes, por ello no fue posible realizar un análisis más fino con el sexo como una variable independiente. Sin embargo, a partir de los datos de dos artículos, se realizó un análisis considerando únicamente a la población femenina que sufrió abuso sexual, y se confirmó la asociación estadísticamente significativa entre la exposición a esta forma de violencia en la infancia y el uso/dependencia por cannabis en las adolescentes, OR 2,22 (IC 95\% [1,86-2,66], Figura 2, panel D).

\section{Discusión}

La investigación científica respecto a las acciones de la cannabis se ha enfocado en su capacidad adictiva y efectos a nivel cerebral; no obstante existen pocos estudios que investiguen la causalidad de la adicción a la cannabis y a otras drogas en sujetos en desarrollo. El hallazgo principal de esta revisión sistemática y metaanálisis es que los adolescentes que han sido violentados en la infancia, mediante el abuso sexual o el abuso físico, tienen mayor riesgo para abusar de la cannabis, o desarrollar dependencia por esta droga.

El análisis estadístico mostró que el abuso sexual fue el predictor más fuerte para el abuso o dependencia de cannabis en adolescentes de uno u otro sexo. En segundo término, el abuso físico ejerció un efecto marginal, pero aún estadísticamente significativo, sobre el abuso o dependencia de la droga; mientras que ser testigo de violencia impactó de forma débil el desenlace principal de esta investigación, mostrando sólo una tendencia estadística. Los OR obtenidos en el análisis estadístico fueron más pequeños que aquellos reportados por Kilpatrick y colaboradores (2000) (abuso físico: OR: 4,84, abuso sexual: OR: 3,80, ser testigo de violencia: OR: 8,42; Tabla 1). Sin embargo, los resultados no se pueden comparar de forma directa ya que, por un lado, el estudio de Kilpatrick et al. (2000) no proporcionó los IC $95 \%$, y por otro, el cuestionamiento sobre la victimización de la violencia se limitó al año anterior a la evaluación, en participantes entre los 12-17 años. En contraste, el análisis efectuado en la presente revisión consideró otros estudios en los cuales la violencia fue ejercida en etapas de la vida más tempranas (antes de los 12 años). Finalmente, la heterogeneidad en las definiciones de la violencia en la infancia podría incidir en los valores del riesgo obtenidos entre ese estudio y el presente trabajo.

Una investigación desarrollada por Caravaca, Navarro, Luna Ruiz-Cabello, Falcon y Luna (2017) en estudiantes universitarios españoles mostró hallazgos similares a los de la presente investigación. Ese estudio incluyó hombres y mujeres con edad promedio de 22,6 años, usuarios de cannabis, los cuales mostraron altas tasas de abuso físico (OR 2,00, IC $95 \%[1,12-3,58]$ ) y abuso sexual (OR 2,72, IC 95\% $[1,06-6,95])$. Los autores usaron un diseño transversal y por ello no analizaron una relación de causalidad entre las variables, ni una dirección para tales eventos (e.g., la victimización sexual incrementa el riesgo de uso de cannabis o el uso de cannabis facilita la victimización sexual). A pesar de estas diferencias, los hallazgos mostrados por Caravaca et al. (2017) dan soporte a la idea de que la exposición a diferentes formas de abuso se relaciona con una mayor vulnerabilidad para el consumo de drogas en la población juvenil, lo que, en conjunto con los datos presentes, subraya la importancia de investigar esta asociación en otras poblaciones a nivel mundial.

Los resultados de los cuatro estudios que analizaron el impacto del abuso sexual sobre el abuso o dependencia por cannabis fueron consistentes, en el sentido de que los valores del intervalo de confianza del $95 \%$ fueron similares, y excedieron el valor de la unidad. Por lo tanto, los resultados del nivel de riesgo (OR) fueron confiables. $\mathrm{Al}$ respecto, el abuso sexual en la infancia duplicó el riesgo de abusar de la cannabis, o desarrollar dependencia por esta droga entre adolescentes de uno u otro sexo. De forma interesante, el abuso sexual a niñas fue un factor crítico para el desarrollo de la dependencia a la droga, aun cuando el número de estudios (dos) y de sujetos (3 885 niñas) analizados fue relativamente bajo. La fortaleza del resultado también es sugerida por la homogeneidad de los reportes, los intervalos de confianza estrechos, y por la significancia estadística de la asociación. Respecto al abuso físico, cuatro estudios reportaron datos para su asociación con el consumo de cannabis. Los resultados mostraron que el intervalo de confianza del $95 \%$ excedió la unidad, sin embargo, fue más amplio que para el abuso sexual, lo cual sugiere un menor impacto del abuso físico sobre el consumo excesivo de cannabis, o el desarrollo de dependencia a esta droga. A su vez, la prueba de heterogeneidad mostró una diferencia estadísticamente significativa cuando se compararon los estudios. La relación entre ser testigo de violencia y el uso de cannabis fue difícil de interpretar, debido al bajo número de estudios (dos) que investigaron esta asociación. Uno de ellos incluyó una muestra muy pequeña, lo que amplificó el intervalo de confianza, y anuló la significancia estadística. A su vez, la prueba de heterogeneidad para los 
datos obtenidos de los testigos de la violencia mostró diferencias significativas entre estos estudios, reforzando la idea de que las poblaciones analizadas no tenían características similares.

El menor impacto del abuso físico y de la exposición indirecta a la violencia sobre el abuso de cannabis, podría ser explicado por las altas tasas de dependencia de la población juvenil por otras sustancias tales como el alcohol (abuso físico: OR: 3,93; ser testigo de violencia: OR: 4,87) y las llamadas "drogas duras" (abuso físico: 12,35; ser testigo de violencia: OR: 13,22) (Kilpatrick et al., 2000). Estos datos son congruentes con lo descrito para usuarios frecuentes de cannabis, o usuarios que iniciaron su consumo en edades tempranas, quienes son más propensos a abusar o desarrollar dependencia por otras drogas respecto a los que consumen cannabis de forma ocasional (de la Fuente et al., 2015).

Los estudios ecológicos, que examinan el contexto en el que se desarrolla el abuso de sustancias, han identificado algunas desventajas sociales y económicas como factores que directamente inciden en las personas expuestas a la violencia: los problemas entre los padres, un apego pobre entre el niño y sus cuidadores primarios, y el uso de sustancias adictivas por parte de los padres, entre otros. A su vez, los factores comunitarios que podrían ser determinantes incluyen el vivir en la pobreza, en vecindarios desorganizados y con acceso fácil a las drogas (Duncan et al., 2008; Fergusson y Lynskey, 1997; Rogosch, Oshri y Cicchetti, 2010). Todos estos factores contribuyen o modulan el uso de sustancias entre individuos que han experimentado una o más formas de violencia (Rogosch et al., 2010). En este contexto, la teoría del afrontamiento (coping) sugiere que los individuos inician el abuso de sustancias en un intento de regular los efectos negativos de la violencia (Foster y Brooks-Gunn, 2009; Harrison, Hoffmann y Edwall, 1989; Kilpatrick et al., 2000; Wright, Fagan y Pinchevsky, 2013). En este sentido, se sabe que las niñas que sufren abuso sexual experimentan desesperanza, problemas de somatización, retraimiento emocional e incluso pueden desarrollar desorden de estrés postraumático. Es importante mencionar que los endocanabinoides participan en la regulación del estrés y una de las acciones agudas de los cannabinoides (por ejemplo, el tetra-hidrocannabinol) es reducir los estados de ansiedad (Zimmermann et al., 2017); por lo tanto, el consumo de cannabis podría regular los estados negativos a través de sus efectos ansiolíticos. Esta interpretación está en línea con la propuesta de que el uso de sustancias es un recurso táctico para regular las emociones y, aunque en controversia, podría representar una forma de automedicación para lidiar con estados emocionales negativos (Khantzian, 1997; Zimmermann et al., 2017).

Desde otra perspectiva, la exposición a la violencia podría conducir al desarrollo de dificultades en el ajuste de la conducta, llevando al individuo a manifestar trastornos o problemas externalizados durante la adolescencia. En correspondencia con esta interpretación, se ha observado una mayor frecuencia de problemas de conducta, alteraciones mentales, delincuencia juvenil, y desórdenes por abuso de sustancias, entre niños maltratados (Fergusson y Horwood, 1998; Fergusson y Lynskey, 1997; Oshri, Rogosch, Burnette y Cicchetti, 2011). De acuerdo con los reportes de la literatura, existen al menos dos escenarios para los adolescentes que consumen drogas: los que usan drogas legales como el alcohol, que es de fácil acceso; y aquellos que, como resultado de alteraciones en su conducta, prefieren una droga ilegal como la cannabis (Oshri et al., 2011; Sartor et al., 2013). En uno y otro caso, el consumo de las sustancias podría llevar a los adolescentes a una victimización o revictimización, lo que podría aumentar el riesgo para el uso futuro de las drogas de abuso, perpetuando de esta manera el ciclo de consumo (Kilpatrick et al., 2000).

La literatura destaca que el uso temprano de cannabis (i.e., durante el periodo crítico del desarrollo cerebral), puede llevar a consecuencias graves y de larga duración (Sartor et al., 2013). A pesar de los argumentos a favor o en contra, la literatura sugiere que el uso crónico de cannabis puede llevar a la adicción (Volkow, Compton y Weiss, 2014). Además, el uso de cannabis ha sido reportado con un factor robusto de riesgo para el subsecuente consumo de otras drogas ilícitas, lo que revela la importancia de la prevención (Fergusson, Boden y Horwood, 2008).

La evidencia muestra que el uso crónico de cannabis afecta el funcionamiento cognitivo, particularmente en aquellos que inician el consumo durante la adolescencia temprana, al reducir de forma significativa el coeficiente intelectual y afectar de manera negativa las funciones ejecutivas, así como la capacidad de atención y la memoria. Este tipo de daño intelectual se ha asociado a una alta deserción escolar y a falta de logros académicos entre los consumidores de cannabis (Ganzer, Broning, Kraft, Sack y Thomasius, 2016; Grant, Gonzalez, Carey, Natarajan y Wolfson, 2003; Volkow et al., 2014). Además, las alteraciones de las funciones motoras que sufren los usuarios de cannabis tienen consecuencias negativas, entre ellas, un aumento en el número de accidentes con vehículos automotores (Volkow et al., 2014). Los datos de la literatura muestran un aumento en el riesgo para padecer psicosis en sujetos con predisposición genética, o una exacerbación de los síntomas psicóticos en pacientes esquizofrenicos (Fergusson, Lynskey y Horwood, 1996; Marconi, Di, Lewis, Murray y Vassos, 2016; Volkow et al., 2014). En relación con otras manifestaciones psiquiátricas, un estudio longitudinal mostró el uso de cannabis como un predictor de violencia interpersonal, así como del desarrollo de trastornos de ansiedad, depresión y algunos trastornos de la personalidad (Copeland, Rooke y Swift, 2013). De forma alarmante, un estudio reciente demostró que el uso crónico y ocasional de cannabis aumentó el riesgo de suicidio entre adolescen- 
tes y adultos jóvenes. El riesgo de suicidio se caracteriza por la presencia de ideación suicida e intentos de suicidio (Borges, Bagge y Orozco, 2016) y se considera el síntoma más grave de varios trastornos psiquiátricos, entre ellos, la depresión (American Psychiatric Association, 2013). En contraposición a la idea, cada vez más difundida, de que el consumo de cannabis conlleva bajos riesgos para la salud (incluida la salud mental), respecto a otras drogas como el tabaco, los hallazgos descritos en la literatura sugieren que esta droga produce graves daños a la salud que podrían reducir de forma importante la calidad de vida.

Los resultados de esta revisión sugieren la importancia de aumentar esfuerzos para prevenir la violencia en la infancia. Asimismo, ponen sobre la mesa de discusión la necesidad de llevar a cabo programas para prevenir el abuso del consumo de cannabis y atender su dependencia, particularmente entre aquellos niños o adolescentes que experimentaron abuso sexual. El consumo repetido de cannabis podría ejercer efectos acumulados sobre la salud y las relaciones del individuo con su entorno familiar y comunitario. El presente estudio subraya la importancia de indagar los antecedentes de violencia en una población, al momento de diseñar los programas de apoyo preventivo para el consumo de drogas de abuso, o las estrategias de intervención. Los individuos con una historia de violencia en la infancia, quienes buscan tratamiento para el consumo descontrolado de cannabis, podrían beneficiarse de intervenciones que favorezcan formas positivas de afrontamiento del estrés, que mejoren su regulación emocional (Vilhena-Churchill y Goldstein, 2014). A su vez, estas intervenciones, además de considerar los efectos farmacológicos de la cannabis, deberían tomar en cuenta el ambiente familiar y comunitario en que vive el afectado, el cual podrían tener ciertos niveles de violencia (Fergusson y Horwood, 1998). El desarrollo de este tipo de programas integrales es urgente, considerando el momento histórico que estamos viviendo donde la despenalización del cultivo y el uso de cannabis en diferentes países ya es una realidad.

\section{Sesgos y limitaciones del estudio}

Una limitación de este estudio fue la calidad de la evidencia mostrada en los artículos que se incluyeron en el metaanálisis, ya que se detectaron varios tipos de sesgos en las investigaciones. El sesgo de selección se infiere a partir de que los estudios originales no adoptaron procedimientos para obtener una muestra aleatoria y representativa de la comunidad. El sesgo de ejecución se presentó por los métodos heterogéneos usados en cada estudio para evaluar la violencia; así mismo, debido a que los trabajos que se evaluaron fueron estudios de seguimiento, los participantes se sometieron a diferentes evaluaciones a lo largo de varias sesiones y para propósitos distintos al objetivo de este artículo (por ejemplo, para obtener información de la heredabilidad del alcoholismo). Es decir, las investigacio- nes no se llevaron a cabo para evaluar exclusivamente el impacto de la violencia sobre el desarrollo de consumo de cannabis. Aunque el aumento de la violencia en general, pero particularmente aquella ejercida hacia los infantes, requiere atención inmediata, y de investigación diseñada exclusivamente para analizar estos determinantes, el diseño de estudios prospectivos con niños como población de estudio podría tener implicaciones éticas, principalmente debido a la exposición a la violencia. Al respecto, los estudios encontrados en la literatura, e incluidos en esta revisión sistemática, fueron de tipo retrospectivo, y el impacto de la violencia en la infancia fue explorado en las etapas finales de la investigación, cuando los participantes se acercaron a la mayoría de edad. Por su parte, el estudio realizado por Dubowitz et al. (2016) describió que los niños en riesgo de abuso y/o de consumo de sustancias, fueron registrados en protocolos para la protección de sujetos de investigación, y referidos a servicios profesionales de atención para infantes (Runyan et al., 1998).

Otra limitación fue el tipo de datos mostrados en cada uno de los artículos, en los cuales no se exploró de forma consistente la gravedad de las formas de violencia, ni su cronicidad. Al respecto, un estudio previo mostró que entre más jóvenes eran los niños al momento de sufrir abuso sexual, el riesgo para consumir cannabis era mayor (Sartor et al., 2013); sin embargo, con los datos obtenidos no pudimos efectuar un análisis de este tipo. Los artículos seleccionados tampoco aportaron evidencia consistente sobre la frecuencia de uso de cannabis, la cantidad consumida, la edad de inicio del consumo, y el tiempo transcurrido entre la primera exposición a la violencia y el uso de la droga, con excepción del estudio de Dubowitz et al. (2016) que incluyó una pregunta sobre el nivel de uso de cannabis (nunca, algún uso, o uso frecuente). Además, no fue posible hacer un análisis de los datos de acuerdo con el sexo de los participantes, y los subtipos de violencia; este tema era de nuestro interés, ya que las encuestas epidemiológicas muestran una mayor frecuencia de consumo de cannabis por niños, respecto a las niñas (United Nations Office on Drugs and Crime, 2015).

En conclusión, los resultados de esta revisión identificaron tipos específicos de violencia ejercida a infantes, tales como el abuso sexual y en menor medida, el abuso físico, como factores que incrementan la vulnerabilidad para el uso o dependencia a cannabis. La exposición a la violencia como un testigo fue un débil predictor del abuso de cannabis. A su vez, el número de estudios que examinaron otros tipos de violencia fue limitado, pero podría ser de interés para el desarrollo de futuros estudios. De hecho, gran parte de la investigación en esta área se enfoca en el impacto del abuso físico o sexual; sin embargo, la negligencia es la forma más común de maltrato, y el maltrato emocional ha sido identificado como un determinante para el desarrollo de psicopatologías (Dubowitz et al., 2016; Vilhena-Chur- 
chill y Goldstein, 2014). Los resultados descritos aquí subrayan la importancia de aumentar esfuerzos para prevenir la violencia, particularmente el abuso sexual, como parte de programas diseñados para reducir el abuso y dependencia por cannabis.

\section{Agradecimientos}

Este trabajo fue financiado por el Grupo Interdisciplinario de Investigación sobre Violencia, Salud Mental y Género del Instituto Nacional de Psiquiatría Ramón de la Fuente Muñiz. Los autores agradecen al Dr. Rodolfo Rivas Ruiz por la revisión crítica de los métodos para llevar a cabo el metaanálisis; a la Dra. Luciana Ramos Lira y Dra. Patricia Fuentes de Iturbe por sus comentarios al manuscrito, a la M. en C. Karla Flores Celis, por el apoyo técnico en el manejo del software RevMan, y a Felipe Campos Gutiérrez por la revisión de estilo de la versión en español.

\section{Financiamiento}

Este trabajo recibió apoyo financiero a través del Programa de Igualdad entre Hombres y Mujeres 2016, del Instituto Nacional de Psiquiatría Ramón de la Fuente Muñiz, y de los proyectos SIP-Instituto Politécnico Nacional.

\section{Contribuciones de los autores}

NPM y LMM desarrollaron el protocolo, extrajeron los datos, y escribieron la primera versión del manuscrito. OTHH y GJR fungieron como revisores independientes del material seleccionado, de acuerdo con los criterios establecidos en la sección de Métodos, y prepararon las tablas y las figuras. Todos los autores leyeron y aprobaron la versión final del manuscrito.

\section{Conflicto de interés}

Los autores declaran que no existen conflictos de interés con las instituciones que dieron el financiamiento.

\section{Referencias}

American Psychiatric Association (1994). Diagnostic and Statistic Manual of Mental Disorders. Fourth edition. Washington, DC: American Psychiatric Publishing.

American Psychiatric Association (2013). Diagnostic and Statistic Manual of Mental Disorders. Fifth edition. Washington, DC: American Psychiatric Publishing.

Barnett, D., Manly, J. T. y Cicchetti, D. (1993). Advances in applied developmental psychology. In D.Cicchetti y S. L. Toth (Eds.), Child Abuse, Child Development, and Social Policy. Norwood, NJ: Ablex Publishing Corporation.
Benjet, C., Borges, G. y Medina-Mora, M. E. (2010). Chronic childhood adversity and onset of psychopathology during three life stages: childhood, adolescence and adulthood. Journal of Psychiatric Research, 44, 732-740. doi:10.1016/j.jpsychires.2010.01.004.

Borges, G., Bagge, C. L. y Orozco, R. (2016). A literature review and meta-analyses of cannabis use and suicidality. Journal of Affective Disorders, 195, 63-74. doi:10.1016/j. jad.2016.02.007.

Buisman-Pijlman, F. T., Sumracki, N. M., Gordon, J. J., Hull, P. R., Carter, C. S. y Tops, M. (2014). Individual differences underlying susceptibility to addiction: Role for the endogenous oxytocin system. Pharmacology, Biochemistry, and Behavior, 119, 22-38. doi:10.1016/j.pbb.2013.09.005.

Caravaca, S. F., Navarro, Z. J., Luna Ruiz-Cabello, A., Falcon, R. M. y Luna, M. A. (2017). Association between bullying victimization and substance use among college students in Spain. Adicciones, 29, 22-32. doi:10.20882/ adicciones.827.

Copeland, J., Rooke, S. y Swift, W. (2013). Changes in cannabis use among young people: impact on mental health. Current Opinion in Psychiatry, 26, 325-329. doi:10.1097/YCO.0b013e328361eae5.

Cottler, L. B. y Compton, W. M. (1993). Advantages of the CIDI family of instruments in epidemiological research of substance use disorders. International Journal of Methods in Psychiatric Research, 3, 109-119.

de la Fuente, J. R., Alvarez Icaza, D., Rodriguez Carranza, R., Ramos Lira, L., Prospéro García, O., Mesa Ríos, F., ... Melgar Adalid M. (2015). Marihuana y Salud. (Primera ed.) Distrito Federal, México: Fondo de Cultura Económica.

Degenhardt, L., Whiteford, H. A., Ferrari, A. J., Baxter, A. J., Charlson, F. J., Hall, W. D., ... Vos, T. (2013). Global burden of disease attributable to illicit drug use and dependence: findings from the Global Burden of Disease Study 2010. Lancet, 382, 1564-1574. doi.10.1016/S01406736(13)61530-5.

Dubowitz, H., Thompson, R., Arria, A. M., English, D., Metzger, R. y Kotch, J. B. (2016). Characteristics of Child Maltreatment and Adolescent Marijuana Use: A Prospective Study. Child Maltreatment, 21, 16-25. doi.10.1177/1077559515620853.

Duncan, A. E., Sartor, C. E., Scherrer, J. F., Grant, J. D., Heath, A. C., Nelson, E. C., ... Bucholz, K.K. (2008). The association between cannabis abuse and dependence and childhood physical and sexual abuse: evidence from an offspring of twins design. Addiction, 103, 990997. doi:10.1111/j.1360-0443.2008.02210.x.

English, D. J., Bangdiwala, S. I. y Runyan, D. K. (2005). The dimensions of maltreatment: introduction. Child Abuse y Neglect, 29, 441-460. doi:10.1016/j.chiabu.2003.09.023.

Fergusson, D. M., Boden, J. M. y Horwood, L. J. (2008). The developmental antecedents of illicit drug use: 
evidence from a 25-year longitudinal study. Drug and Alcohol Dependence, 96, 165-177. doi:10.1016/j.drugalcdep.2008.03.003.

Fergusson, D. M. y Horwood, L. J. (1998). Exposure to interparental violence in childhood and psychosocial adjustment in young adulthood. Child Abuse $\mathcal{E}$ Neglect, 22, 339-357. doi/10.1016/S0145-2134(98)00004-0.

Fergusson, D. M., Horwood, L. J. y Lynskey, M. T. (1996). Childhood sexual abuse and psychiatric disorder in young adulthood: II. Psychiatric outcomes of childhood sexual abuse. Journal of the American Academy of Child and Adolescent Psychiatry, 35, 1365-1374. doi:10.1097/00004583199610000-00024.

Fergusson, D. M., Horwood, L. J., Shannon, F. T. y Lawton, J. M. (1989). The Christchurch Child Development Study: a review of epidemiological findings. Paediatric and Perinatal Epidemiology, 3, 302-325. doi:10.1111/j.1365-3016.1989.tb00382.x.

Fergusson, D. M. y Lynskey, M. T. (1997). Physical punishment/maltreatment during childhood and adjustment in young adulthood. Child Abuse E Neglect, 21, 617-630. doi:10.1016/S0145-2134(97)00021-5.

Fergusson, D. M., Lynskey, M. T. y Horwood, L. J. (1996). Childhood sexual abuse and psychiatric disorder in young adulthood: I. Prevalence of sexual abuse and factors associated with sexual abuse. Journal of the American Academy of Child and Adolescent Psychiatry, 35, 1355-1364. doi:10.1097/00004583-199610000-00023.

Folkman, S. y Lazarus, R. S. (1988). Coping as a mediator of emotion. Journal of Personality and Social Psychology, 54, 466-475. doi:10.1037/0022-3514.54.3.466.

Folkman, S., Lazarus, R. S., Gruen, R. J. y DeLongis, A. (1986). Appraisal, coping, health status, and psychological symptoms. Journal of Personality and Social Psychology, 50, 571-579. doi:10.1037/0022-3514.50.3.571.

Foster, H. y Brooks-Gunn, J. (2009). Toward a stress process model of children's exposure to physical family and community violence. Clinical Child and Family Psychology Review, 12, 71-94. doi:10.1007/s10567-009-0049-0.

Ganzer, F., Broning, S., Kraft, S., Sack, P. M. y Thomasius, R. (2016). Weighing the evidence: A systematic review on long-term neurocognitive effects of cannabis use in abstinent adolescents and adults. Neuropsychology Review, 26, 186-222. doi:10.1007/s11065-016-9316-2.

Grant, I., Gonzalez, R., Carey, C. L., Natarajan, L. y Wolfson, T. (2003). Non-acute (residual) neurocognitive effects of cannabis use: a meta-analytic study. Journal of the International Neuropsychological Society, 9, 679-689. doi:10.1017/S1355617703950016.

Harrison, P. A., Hoffmann, N. G. y Edwall, G. E. (1989). Differential drug use patterns among sexually abused adolescent girls in treatment for chemical dependency. The International Journal of the Addictions, 24, 499-514. doi:10.3109/10826088909081832.
Jester, J. M., Steinberg, D. B., Heitzeg, M. M. y Zucker, R. A. (2015). Coping expectancies, not enhancement expectancies, mediate trauma experience effects on problem alcohol use: A prospective study from early childhood to adolescence. Journal of Studies on Alcohol and Drugs, 76, 781-789. doi:10.15288/jsad.2015.76.781.

Kessler, R. C., McLaughlin, K. A., Green, J. G., Gruber, M. J., Sampson, N. A., Zaslavsky, A. M., ... Williams D.R. (2010). Childhood adversities and adult psychopathology in the WHO World Mental Health Surveys. The British Journal of Psychiatry, 197, 378-385. doi:10.1192/bjp. bp.110.080499.

Khantzian, E. J. (1997). The self-medication hypothesis of substance use disorders: a reconsideration and recent applications. Harvard Review of Psychiatry, 4, 231-244. doi:10.3109/10673229709030550.

Kilpatrick, D. G., Acierno, R., Saunders, B., Resnick, H. S., Best, C. L. y Schnurr, P. P. (2000). Risk factors for adolescent substance abuse and dependence: data from a national sample. Journal of Consulting and Clinical Psychology, 68, 19-30. doi:10.1037/0022-006X.68.1.19.

Kuhar, M. (2012). The Addicted Brain. Why we abuse drugs, alcohol, and nicotine. Upper Saddle River, NJ: FT Press.

Marconi, A., Di, F. M., Lewis, C. M., Murray, R. M. y Vassos, E. (2016). Meta-analysis of the association between the level of cannabis use and risk of psychosis. Schizophrenia Bulletin, 42, 1262-1269. doi:10.1093/schbul/sbw003.

Meier, M. H., Caspi, A., Ambler, A., Harrington, H., Houts, R., Keefe, R. S., .. Moffitt, T.E. (2012). Persistent cannabis users show neuropsychological decline from childhood to midlife. Proceedings of the National Academy of Sciences of the United States of America, 109, E2657-E2664. doi:10.1073/pnas.1206820109.

Oshri, A., Rogosch, F. A., Burnette, M. L. y Cicchetti, D. (2011). Developmental pathways to adolescent cannabis abuse and dependence: child maltreatment, emerging personality, and internalizing versus externalizing psychopathology. Psychology of Addictive Behaviors, 25, 634644. doi:10.1037/a0023151.

Osofsky, J. D. (1999). The impact of violence on children. Future Child, 9, 33-49. doi:10.2307/1602780.

Ramaekers, J. G., Kauert, G., van, R. P., Theunissen, E. L., Schneider, E. y Moeller, M. R. (2006). High-potency marijuana impairs executive function and inhibitory motor control. Neuropsychopharmacology, 31, 2296-2303. doi:10.1038/sj.npp.1301068.

Rogosch, F. A., Oshri, A. y Cicchetti, D. (2010). From child maltreatment to adolescent cannabis abuse and dependence: a developmental cascade model. Development and Psychopathology, 22, 883-897. doi:10.1017/ S0954579410000520.

Runyan, D. K., Curtis, P. A., Hunter, W. M., Black, M. M., Kotch, J. B., Bangdiwala, S. I., ... Landsverk, J. (1998). 
Longscan: A consortium for longitudinal studies of maltreatment and the life course of children. Aggression and Violent Behavior, 3, 275-285. doi:10.1016/S13591789(96)00027-4.

Sartor, C. E., Agrawal, A., Grant, J. D., Duncan, A. E., Madden, P. A., Lynskey, M. T., ... Bucholz, K.K. (2015). Differences between african-american and european-american women in the association of childhood sexual abuse with initiation of marijuana use and progression to problem use. Journal of Studies on Alcohol and Drugs, 76, 569577. doi:10.15288/jsad.2015.76.569.

Sartor, C. E., Waldron, M., Duncan, A. E., Grant, J. D., McCutcheon, V. V., Nelson, E. C., ... Heath, A.C. (2013). Childhood sexual abuse and early substance use in adolescent girls: the role of familial influences. Addiction, 108, 993-1000. doi:10.1111/add.12115.

Spear, L. P. (2000). The adolescent brain and age-related behavioral manifestations. Neuroscience and Biobehavioral Reviews, 24, 417-463. doi:10.1016/S0149-7634(00)00014-2 Spear, L. P. (2013). Adolescent neurodevelopment. The Journal of Adolescent Health, 52, S7-13. doi:10.1016/j.jadohealth.2012.05.006.

Substance Abuse and Mental Health Services Administration. (2015). Behavioral Health Barometer. Rockville, MD, Substance Abuse and Mental Health Services Administration. Recuperado de https://www.samhsa.gov/ data/sites/default/files/2015_Maryland_BHBarometer.pdf.

United Nations Office on Drugs and Crime. (2015). World Drug Report 2015. New York, United Nations publication, Sales No. E.15.XI.6. Recuperado de https://www. unodc.org/documents/wdr2015/World_Drug_Report_2015.pdf.

Vilhena-Churchill, N. y Goldstein, A. L. (2014). Child maltreatment and marijuana problems in young adults: examining the role of motives and emotion dysregulation. Child Abuse E̋ Neglect, 38, 962-972. doi:10.1016/j. chiabu.2013.10.009.

Villatoro-Velázquez, J. A., Medina-Mora, M. E., Fleiz-Bautista, C., Téllez-Rojo, M. M., Mendroza-Alvarado, L. R., Romero-Martínez, M., ... Guisa-Cruz V. (2012). Encuesta Nacional de Adicciones 2011: Reporte de Drogas. México D.F., México, Instituto Nacional de Psiquiatría Ramón de la Fuente Muñiz; Instituto Nacional de Salud Pública; Secretaría de Salud. Recuperado de http://inprf.gob. $\mathrm{mx} /$ psicosociales/archivos/ena/ena2011_drogas.pdf.

Volkow, N. D., Compton, W. M. y Weiss, S. R. (2014). Adverse health effects of marijuana use. The New England Journal of Medicine, 371, 879. doi:10.1056/NEJMra1402309.

World Health Organization. (2016). Management of substance abuse. Cannabis. 5-9-2016. Recuperado de http:// www.who.int/substance_abuse/facts/cannabis/en/.

Wright, E. M., Fagan, A. A. y Pinchevsky, G. M. (2013). The effects of exposure to violence and victimization across life domains on adolescent substance use. Child Abuse $\mathcal{E}^{2}$ Neglect, 37, 899-909. doi:10.1016/j.chiabu.2013.04.010.

Zimmermann, K., Walz, C., Derckx, R. T., Kendrick, K. M., Weber, B., Dore, B., ... Becker, B. (2017). Emotion regulation deficits in regular marijuana users. Human Brain Mapping, 38, 4270-4279. doi:10.1002/hbm.23671. 